\title{
Analysis on the Regularity of Gynecological External Treatment Liya $\mathrm{Ma}^{1, \mathrm{a}}$, Zhenghui Chang ${ }^{1, \mathrm{~b}}$ \\ ${ }^{1}$ Henan University of Traditional Chinese Medicine, Zhengzhou, Henan, 450046 \\ ${ }^{\mathrm{a}}$ email, ${ }^{\mathrm{b}}$ email
}

Keywords: The Regularity, Gynecological External Treatment, Medicine

\begin{abstract}
This article collects and arranges prescriptions of gynecological diseases in ancient physicians, sums up its rules of prescriptions, uses range of prescriptions, formulas and usage of prescriptions and finds and selects high frequency prescriptions and drugs. In order to better guide the clinical use of drugs, and for the gynecological disease outside the provision of new ideas for future research to provide a large number of concentrated literature resources and theoretical data.
\end{abstract}

\section{Introduction}

Gynecological medicine external treatment has the external, outward or superficial parts of the drug, or deposited or smoked or washing, drug penetration through the skin or membrane absorption play a role. In most cases, for the treatment of superficial disease, but sometimes in the body surface treatment, but also treatment of internal organs diseases, such as umbilical therapy. At present, the study of Chinese medicine gynecological disease treatment focused on the clinical side, the lack of traditional books, literature research and finishing, few prescriptions of gynecological diseases with prescriptions to explore; therefore, the gynecological disease prescription side features Get detailed comments, affecting the maximum effect of Chinese medicine to play.

\section{Chinese Medicine Gynecological Topical Prescription Range of Disease}

Before the Tang: the diseases involved are as follows: menstrual disease: water adverse .Pregnancy disease: fetal death, pregnancy heartache, pregnancy disease, adverse health .Postpartum disease: postpartum hot junction stool unreasonable postpartum virtual cold Yumen closed, woman Breast disease: nipple break, breast abscess and nipple itching. Yin before the disease: Yin sore, Yin pain, Yin Yang, cervical polyp, Yin blowing, Yin wide, cold, Yin Gynecological miscellaneous diseases: women and ghosts, yin and yang noir pain can not be tolerated, no child, the woman will never be born, about 26 diseases.

Song Jin Yuan: menstrual disease: women blood lame, dysmenorrhea. Pregnancy disease: Prophylaxis does not open, pregnancy typhoid fever injury fetal damage, pregnancy urination barrier. Postpartum disease: post-natal Yin pain. Milk: breast sores, milk carbuncle, woman breast. A Yin disease: Yin swelling does not accept, Yin swollen pain, Yin cold itching, Yin Tong sores, Yin carbuncle, Yin off, Yin erosion, Yin Ting. Gynecological miscellaneous diseases: women's blood tumor, the woman chill no child, the woman sub-dirty air, the woman must never return, partial wind, head wind pain, urination bleeding, hemorrhoids, prolapse, about 25 diseases.

Ming and Qing Dynasties: menstrual disease: uterine bleeding, amenorrhea and Chibai vaginal discharge, dysmenorrhea, menstrual premenstrual volume, irregular menstruation, menstrual caking pain. Vaginal discharge: under the damp, vaginal discharge, Chibai vaginal discharge. Pregnancy disease: pregnant women, a pox fetal movement, fetal movement disturbed, under the stillbirth, delayed production, fetal movement disturbed, pregnancy. Postpartum stroke, postpartum stroke, the door does not close, maternal intestine does not close, postpartum Zhu Zheng, postpartum blood Jue, postpartum hemorrhage, postpartum hemorrhage, Maternal Dan language palpitation, Jing palpitations, do not close the doors and throat. Milk: nipple rupture, breast abscess, breast, breast, milk, milk, milk, milk, milk, milk. Yin disease: Yin sore, Yin Tong, temple addiction, vaginal swelling and pain, Yin Yang, Yin Ting, Yin cold, swollen vulva, sore wet sores, gynecological miscellaneous diseases: addiction, women blood knot chest, Blood clotting, blood poisoning, 
urinary heat closed, consumer product line fatigue, Zhuji, fat addiction, small households to marry pain, infertility, blood halo, about 61 diseases.

Modern and modern: vaginal disease: vaginal discharge. Pregnancy disease: vicious resistance. Postpartum disease: postpartum Yin pain. Vaginal bleeding, Yin Ting, pubic lice, palace Mi, Behcet's syndrome, genital alopecia, female vaginal leukoplakia, Yin Yin, Yin Yin, , Yin-sore into repeated, heat poison internal sense, congestion heat, uterine prolapse. Gynecological diseases: slow pelvic inflammatory disease, fallopian tube disorders, hair loss about 25 diseases. Overview of the above diseases has the liver through the main parts.

There are (4.10\%), vaginal itching (4.12\%), vaginal pain (4.120\%), vaginal pain $(1.76 \%)$ and postpartum stroke (1.47\%). These eight high frequency diseases accounted for $41.47 \%$ of the total number of diseases treated by gynecological diseases.

\section{Chinese Medicine Gynecological Topical Prescription Formulations}

Different formulations due to the production, use and the role of different characteristics, and thus have their own personality characteristics, observe the preparation process of various formulations, including the most complex plaster, such as 5 Taiping Sheng Hui Fang 6 in the preparation of fish paste side: Fried lard so that the boiling, that is, under the fried fish smoke, the next issue of the order pin, filter sediment, under the realgar yellow end of the sulfur, stir to make uniform, Sheng porcelain, followed by powder, but also useful vinegar, wine and other different substrates (Accounting for 31.47\%), suppositories (21.47\%), ointment (17.94\%), powder (11.76\%), the mechanism of action of the ancients have (see Table 6), the treatment of gynecological diseases, Has been described, can be divided into from the skin penetration, or through the pay film absorption, and conduction through the meridian. Such as Xu Dachun in the 5 source of medical theory 6 on the plaster mechanism of the elaboration, that paste with the paste, the occlusion of the gas.

\section{Chinese Medicine Gynecological Topical Prescription Usage}

Fumigation method: in order to achieve swelling, pain, itching and soften the local tissue of the purpose of the decoction of the liquid in the vulva fumigation, washing and bathing. Chinese medicine with cold water immersion is after about 90 minutes, filter out the liquid into the container, while hot fumigation affected area, to be slightly cool again wash the affected area and bath treatment.

Flushing method: for the treatment of Yin Yang, vaginal discharge and other symptoms and more syndromes, the liquid directly to the vulva, vagina and achieve therapeutic purposes. However, vaginal congestion or ulceration is prohibited. About 370 with the following liquid $500 \mathrm{ml}$, into the disposable vaginal washing device, according to the routine operation of disinfection rinse.

Satisfied drug method: for the treatment of dampness itching, detoxification insecticide, remove rot myogenic and contraction of the uterus and other therapeutic purposes, the drug into the vagina or bend to direct the role in the local.

Enema method (anal introduction method): for the treatment of pelvic inflammatory mass, chronic pelvic inflammatory disease and pelvic addiction blood syndrome, the decoction of traditional Chinese medicine concentrated poured into the rectum retention, to play the efficacy. Because the role of local (rectal and pelvic reproductive system close to), while the rectal wall venous plexus rich, easy to absorb the active ingredients of drugs.

Intrauterine injection: made of traditional Chinese medicine injection, routine disinfection of the vulva, vagina, cervix, the liquid into the uterine cavity and fallopian tubes, for therapeutic diagnosis.

Chinese medicine gynecological external prescriptions prescription medication

Ancient physicians have attached importance to heat detoxification, astringent class of sores drugs, mainly in heat-based drugs. Commonly used drugs are Cork, Rong basin, Patrinia, Coptis, Huang Qin and so on. It is often compatibility insecticidal antipruritic drugs, in order to insecticidal 
itching effect.

Attack insecticidal drugs to detoxification treatment sores, attack insecticide, dampness itching as the main role, most have different degrees of toxicity, should be strictly controlled dosage and usage, not excessive and continuous use, to prevent poisoning. Such as high-frequency drugs: Cnidium, alum, realgar, sulfur and so on. Relieving the spread of drugs in the epidermis, defenses against exogenous pathogens invasion; ancient master common understanding of the type of solution table Drugs, with open-mouth fur, catharsis skin, through the evil out of the effect. Blood circulation addiction drugs to smooth blood, dissipate addiction blood as the main role, Wei Du Xin, bitter, the main return of the liver, heart, into the blood, through the blood of fatigue, resulting in pain, menstruation, Swelling, eliminate blood carbuncle and so on. Fatigue is a pathological product, but also a variety of disease pathogenic factors. For the formation of addiction blood of different etiology and disease, it is with the compatibility of the standard wood to both. Wensi class of drugs and more warm and warm, with its Xinsan temperature, partial organs and can be warm and cold, warm through the pain.

Five to Xin, bitter, Gan accounted for $83.13 \%$ of the three drugs accounted for. Because each drug has both gas and taste, so the two must be integrated together to see; Miao Xi Yong said: the gas has to taste, there are gas and sexual. Emphasizing the medicine is composed of gas and taste together, that must be combined with the four gas and flavors, Diao an accurate identification of the role of drugs; which can complement, can slow, and energy, that is, replenishing righteousness, enhanced Physical, in order to enhance the role of disease resistance; Xin: to San, can run, can do, there is divergence, qi, blood and other functions; bitter: to vent, can dry, can Jianyin (Xiehuo yin) Expelling heat, venting gas inverse, venting stool, dampness and other effects; ancient physicians are Xin Wen divergence, open the skin through the first choice of compatibility of the evil out of the evil, temperature and Xinju four gas flavors of medication first, so far is also the most Basic clinical treatment of gynecological diseases topical prescriptions principles; according to Chinese medicine theory pointed out that in addition to Xin Wen, the bitter cold is also the treatment of gynecological diseases of the main method; temperature can dispel cold, Gan Hanqing heat is not Shangyin and Sheng Jin, Gan Wen drugs With the role of qi to help yang, bitter cold can heat dampness. Bitter drop to wet by, spicy to remove foul, cold take almost gas, by gas line, not occlusion in the also, for the gynecological disease of damp heat evil mixed, Addiction and not loose, for a long time because of poison. Commonly used in this study Xinwen drugs Cnidium, Chuanjiao, etc., commonly used bitter cold medicine rhubarb, Pu nitrate and so on.

Lung drugs: the lungs of the main gas, the Secretary for breathing, the main Xuanfa Su drop, external coats, opens into the nose. The lungs of the hair function to make the water grain distribution of fine grain in the hair, nourishing the whole body skin, hair, muscle; physicians also respected lung medicine, due to the initial evil stem lung health to light Qingzhi for the rule; medication such as high frequency alum , Dry alum, Angelica, almond, yellow answer, one hundred, yellow, forsythia, Nepeta, wind, Scrophulariaceae, cicada sloughing; If lung qi, evils easily invaded, , If the lungs by the heat, can cause itching; head _ lung and large intestine surface, the lungs declared declining, the impact of large intestine conduction dross function. Spleen medicine: spleen medicine is also respected by physicians, because the spleen and stomach for the day after tomorrow, the source of qi and blood biochemistry, metaplasia to irrigation Quartet subtle. The spleen of the main transport, the main gas, the gas main $\mathrm{l}$ is with a unified blood, solid-uterus of the right. The stomach is the main receiving, for the water valley of the sea, is more gas and more blood of the internal organs, foot Yangming and Chongmai will be in the air street, it is under the Yang Ming said.

Topical prescriptions are also more common in herbal medicine Mingxian eighteen against the use of fear of anti-drug, both for topical drugs, may not cause great harm to the human body; and different seek the same, that the use of fear of anti-drug Some characteristics, which need further study.

The frequency of poison has reached 570\%, indicating that there are drugs in the topical treatment of gynecological diseases, played a role in the toxic drugs are anti-inflammatory 
insecticidal itching realgar, sulfur, alum, Cnidium, etc.; Light powder; deworming Chung spit of the acacia pods; there is no lack of Guanzhong; diarrhea under the Croton, Kansui; search the wind Scorpio, enter clams; rheumatism of the grass Ukraine, Chuanwu: According to the principles of traditional Chinese medicine to poisoning attack, the treatment; In addition, the ancient emphasis on external drug processing, preparation of the preparation, in the production of almonds, almond, almond, Arisa, Pinellia; temperature of the Wu Zhu Ying, Asarum, In the process, the toxicity of toxic drugs will decrease or even disappear. .

\section{Conclusion}

Gynecological topical prescriptions of traditional Chinese medicine is with a wide range of applications, including a variety of gynecological vaginal delivery with a variety of syndromes, reflecting the gynecological genital disease of rheumatic fever drug stagnation, blood deficiency syndrome and the convergence of the disease outside the treatment of thought. Chinese medicine gynecological topical prescription dosage forms and the use of a wide range of time due to etiology, selects of different formulations and usage according to the different types of disease and stage, in which the acute disease to lotion, suppository, powder, powder, chronic recurrent disease syndromes, Suppository-based; usage to wash method, satisfied that France, blending method for more. Dialectical study of the above prescriptions and it will play a role prevention and treatment of modern gynecological diseases.

\section{References}

[1] Check JH, Choe JK, Lee G, DietterichC. The effect on IVF outcome of small intramural fibroids not compressing the uterine cavity as determined by a prospective matched control study. Human Reproduction. 2002

[2] Dietterich C, Check JH, Choe JK, Nazari A, Fox F.The presence of small uterine fibroids not distorting the endometrial cavity does not adversely affect conception outcome following embryo transfer inolder recipients. Clin Exp Obstet Gynecol. 2000

[3] Dubuisson JB, Chapron C, Verspyck E, et al.Laparoscopic myomectomy: 102 cases. Contrac Fertil Sexual . 1993

[4] Hasson HM,Rotman C,Rana N, et al. Laparoscopicmyomectomy. Obstetrics and Gynecology. 1992

[5] Nezhat C, Nezhat F, Silfen SL, et al. Laparoscopicmyomectomy. International Journal of Fertility. 1991 